\title{
Cosmic Ray Associated with Coronal Index and Solar Flare Index during Solar Cycle 22 - 23
}

\author{
Prithvi Raj Singh, Shabir Ahmad, A. C. Pandey, Ajay Kumar Saxena, Chandra Mani Tiwari, \\ A. P. Mishra \\ Department of Physics, A.P.S. University, Rewa, M.P., India \\ Email: id-prithvisingh77@gmail.com
}

How to cite this paper: Singh, P.R., Ahmad, S., Pandey, A.C., Saxena, A.K., Tiwari, C.M. and Mishra, A.P. (2017) Cosmic Ray Associated with Coronal Index and Solar Flare Index during Solar Cycle 22 - 23. International Journal of Astronomy and Astrophysics, 7, 162-173.

https://doi.org/10.4236/ijaa.2017.73013

Received: June 18, 2017

Accepted: August 2, 2017

Published: August 5, 2017

Copyright $\odot 2017$ by authors and Scientific Research Publishing Inc. This work is licensed under the Creative Commons Attribution International License (CC BY 4.0).

http://creativecommons.org/licenses/by/4.0/

\begin{abstract}
We study the relation between monthly average counting rates of the cosmic ray intensity (CRI) observed at Moscow Neutron Monitoring Station, solar flare index (SFI) and coronal index during the solar cycles 22 and 23, for the period 1986-2008. The long-term behaviour of various solar activity parameters: sunspot numbers (SSN), solar flare index $\left(\mathrm{H}_{\alpha}\right.$ flare index), coronal index (CI) in relation to the duration of solar cycles 22 and 23 is examined. We find that the correlation coefficient of CRI with the coronal index as well as $\mathrm{H}_{\alpha}$ flare index is relatively large anti-correlation during solar cycle 22. However, the monthly mean values of sunspot number, $\mathrm{H}_{\alpha}$ flare index, and coronal index are well positively correlated with each other. We have analyzed the statistical analysis of the above parameters using of linear model and second order polynomial fits model.
\end{abstract}

\section{Keywords}

Solar Activity, Sunspot Number, Coronal Index, Solar Flare Index, Cosmic Ray Intensity

\section{Introduction}

In the past, a lot of work has been done on the correlation of solar parameters with the intensity of cosmic ray on the long-term basis. The source of solar activity is the variation of the solar magnetic field, which is usually manifested by sunspots on the solar surface. They called cyclic variation of 11-years of sunspot numbers (SSN) was first recognized [1]. The most obvious indicators of solar activity are sunspots, flares, plages, and so on [2]. The numbers of sunspots were generally used as a reliable parameter to measure solar activity [3] [4] [5]. The 11-year's variation in the intensity of cosmic ray observed on the 
Earth is found anti-correlated with the variation in number of sunspots [6]. The solar modulation of cosmic ray intensity has been studied in recent decades using data from ground based detectors, in combination with various solar activity and solar-terrestrial relations [7] [8] [9] [10] [11]. Galactic cosmic rays in the energy range from several hundreds $\mathrm{MeV}$ to tens of $\mathrm{GeV}$ are subjected to heliospheric modulation, under the influence of solar output and its variation. The heliospheric modulation of the cosmic ray intensity and spectrum are associated with the 11-years of solar activity cycle. The polarity dependence on the drift mechanism is clearly observed in the cosmic ray modulation in terms of 22-years of solar magnetic cycle, showing different shapes of cosmic ray intensity. In the long-term modulation of cosmic ray in the high energy range is studied using the monthly average date of global network neutron monitoring stations of cosmic ray with different geomagnetic cut-off rigidities [12] [13].

A $\mathrm{H}_{\alpha}$ flare index (SFI) is a short-lived phenomenon observed as sudden increases in the intensity of the radiation emitted in the neighbourhood of sunspots. While observing the Sun projected on a screen, [14] [15] noted that $\mathrm{H}_{a}$ flare index lasted about $\sim 5$ minutes from many ground-based observatories and characterizations had been made [16]. $\mathrm{H}_{\alpha}$ flare indexes are powerful bursts of solar activity that occurs on time scales ranging from tens of minutes to a few hours and can produce a series of the Sun-Earth effects [17]. The solar flare activity appears closely are correlated with sunspots and major eruptions often seen near the largest and most complex active regions [18]. Recently, the SFI has been reported as one of the best solar parameters to explain the heliospheric physical changes with respect to time [19]. Coronal Index (CI) represents the total irradiance of the Sun through the $530.3 \mathrm{~nm}$ of coronal emission lines as that observed by means of the ground-based coronagraph worldwide [20] [21] [22]. Recent works in [23] [24] found an empirical relationship between the coronal index of solar activity, the number of sunspots and solar flare grouped for the period 1966-1998. In this report, an expressed by the contribution of the solar magnetic field has been attributed in order to find a better accuracy between the observed values and the calculated values of the coronal index time series. This index is the total irradiance of green line corona in the visible solar hemisphere and possibly reflects the distribution of photospheric magnetic fields in the solar corona. The variation in the intensity of cosmic ray is attributed to changes in the solar wind structure and the interplanetary magnetic field in the solar source variability [25] [26] [27]. The behaviour of the solar wind and plas$\mathrm{ma}$ in the interplanetary medium is significantly associated to strong solar flares represented by the $\mathrm{H}_{\alpha}$ flare index (SFI).

In the present paper, we have studied the relative merits of various solar indices (SSN, SFI and CI) in relation to CRI. We have adopted (Linear model and Polynomials of second order model) to analyze and correlate the characteristics of solar activity and cosmic ray intensity during the solar cycles $22-23$. 


\section{Data Analysis}

In this analysis, the monthly variation of cosmic ray intensity (CRI) in association with solar activity: Sunspot Number (SSN), Coronal Index (CI) and $\mathrm{H}_{\alpha}$ flare index (SFI). The correlation coefficients have been calculated between Cosmic Ray Intensity (CRI) with SFI and CI, SSN with SFI and CI. We have analyzed the interconnection between various parameters CRI, CI, SFI and SSN with the help of the approximation by the polynomials of second order.

Cosmic Ray Intensity (CRI): Cosmic rays actually are not rays at all but particles, it consist $90 \%$ of protons, and $9 \%$ of alpha particles. The hourly pressure-corrected of cosmic ray intensity are available different places of Neutron Monitoring Station at different cut-off rigidities. In the present study, we have used the monthly mean cosmic ray intensity from high latitude stations situated at Moscow, with cut-off rigidities $(2.43 \mathrm{GV})$ taken from data

(http://cr0.izmiran.rssi.ru/mosc/main.htm).

$\mathrm{H}_{a}$ flare index (SFI): In order to quantify the daily (H-alpha) flare activity over 24 hours per day [28] introduced to the 'flare index' is defined as $\mathrm{Q}=\mathrm{i} \times \mathrm{t}$, where "i" represents the intensity scale of importance to flare observed in $\mathrm{H}_{\alpha}$ and " $\mathrm{t}$ " the duration (in minutes) of the flare and " $\mathrm{Q}$ " denotes total energy emitted by solar flare [16]. The $\mathrm{H}_{\alpha}$ flare index taken from data

(http://www.ngdc.noaa.gov/stp/space-weather/solar-data/solar-features/solar-flare s/index).

Sunspot number (SSN): Historically, the relative sunspot number " $R$ " is the most basic indicator of solar activity. Each isolated cluster of sunspots is called a group of sunspot, and it can consist of one or many distinct spots. The relative number of sunspot is defined as $R=K(10 g+f)$, where " $g$ " is the number of identified sunspot groups, "f" is the number of individual sunspots and " $\mathrm{K}$ " is a correction factor of the observer. The sunspot number is taken from (http://www.ngdc.noaa.gov/stp/spaceweather/solardata/solarindices/sunspotnu mbers).

Coronal index (CI): The Coronal index (CI) is represents an average daily power (irradiance) emitted in the green line (Fe XIV) at $530.3 \mathrm{~nm}$ wavelengths. It is the total energy emitted by the Sun of the outermost atmospheric layer, which is expressed in $10^{16} \mathrm{~W} / \mathrm{Sr}$ and is taken from

(http://www.ngdc.noaa.gov/stp/space-weather/solar-data/solar-indics/solar_cor ona/coronal-index/slovak).

\section{Results and Discussion}

\subsection{Variations of CRI with CI and SFI}

In Figure 1, we have presented time evolution of monthly mean variations of CRI with CI and SFI is inversely correlated for the period 1986-2008. The maximum of SFI and CI does not coincide with the exact minima of the CRI during solar cycles 22 - 23. We have noted that, time-lags for smooth variation ( 6-Months) between CI and CRI, for maximum to minimum during solar cycle 
22, A-a ( 6 Months (Aug. 1989 to Jan. 1990)), and for solar cycle 23, C-e ( 18 Months (Aug. 2000 to Dec. 2001)), as well as for minimum to maximum in solar cycle 23, B-d ( 11 Months (Aug. 1996 to June 1997)). SFI and CRI, for maximum to minimum during solar cycle 22 is A-a ( 6 Months (Aug. 1989 to Jan. 1990)), C-c ( 4 Months (April 1991 to July 1991)), and for minimum to maximum in solar cycle 22 is B-b ( 7 Months (July 1990 to Jan. 1991)), and for solar cycle 23, D-d ( 7 Months (May 1997 to Nov. 1997)). We have observed the period of Oct. 1999 to Dec. 2003 (line 3 to 4) in SFI with CRI is not prominent (anti-phase) as well as CI with CRI is minimum anti-phase during the solar cycle 23 (see Figure 1).

Therefore, we have concluded the phase-lags between CRI and CI is larger than the CRI with SFI (see Figure 1). We have observed smooth variation of SFI is two peaks (A and C) and one minimum (B) and CRI is two minimum (a and c) and single peak (b) in solar cycle 22 is strong ant-phase as well as for solar cycle 23 is same pattern, for SFI is maximum ( $E$ and G), and minimum (F) and CRI is two maximum ( $d$ and $f$ ) and two minimum (e and g). Solar cycle 23 is minimum anti-phase (lowest amplitude) as compare to solar cycle 22. We have pointed out there is a high level of flare activity in the solar cycle 22 and very low level of flare activity in the solar cycle 23 . In the solar cycle $22-23$, the coronal index is more efficiently generated power (irradiance) emitted in the green line
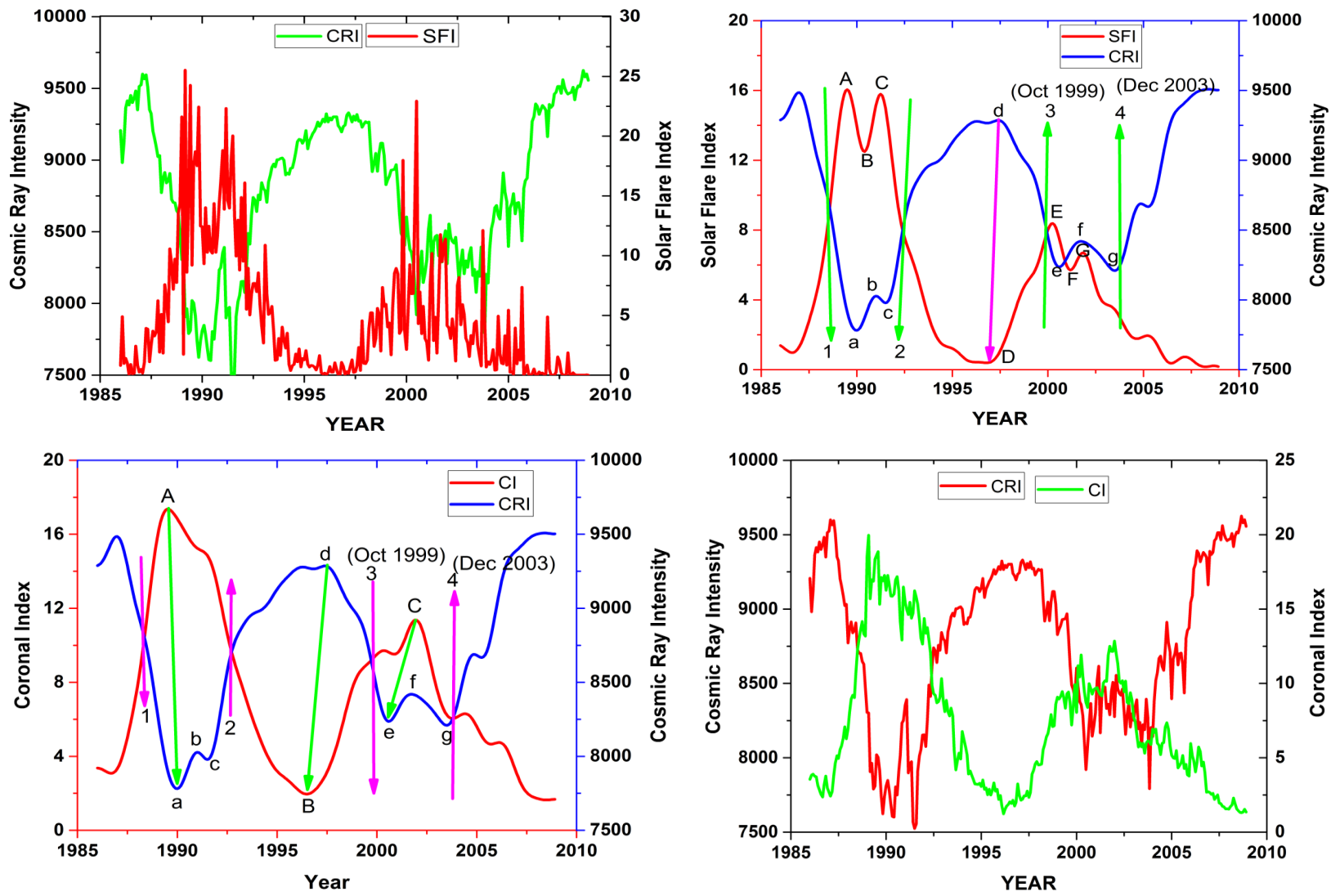

Figure 1. Time profiles of the monthly average values of CRI with CI (upper left panel) and CRI with SFI (lower left panel), and smooth variation ( 6-Months) CRI with CI (upper right panel) and CRI with SFI (lower right panel) during Solar Cycles 22 and 23. 
(Fe XIV) at $530.3 \mathrm{~nm}$ wavelengths during the maximum, and it appears that production is declining toward the end of the cycle.

\subsection{Variations of SSN with CI and SFI}

We have observed that there is a close correspondence in the long-term variation of SSN and CI, where CI is closely tracks the SSN at all the time during solar cycle 22 and 23, and peak values is larger for cycle 22 as compare to cycle 23 . The significant change in the amplitude and behaviour of Coronal Index (CI) for solar cycles 22 and 23 are observed in accordance with the SSN. The differences between peak values of SSN and SFI are also found to continuously decrease for solar cycles 22 to 23 (see right panel of Figure 2). The SFI is closely same track the SSN for solar cycle 22 - 23. [29] proposed that, the high level of solar activity in the cycle 21 and very low level of solar activity in the cycle 23 . In the smooth variation ( $\sim 6$-Months) of solar cycle 22 and 23, two peaks on the SSN and single peak of CI and no time-lags are present. We have observed the northern and southern hemisphere is present and there is no time-lag between maximum to minimum between SSN and SFI during the solar cycles 22 and 23. In this study, solar wind plays an important role at the high level of amplitude for SSN, CI and
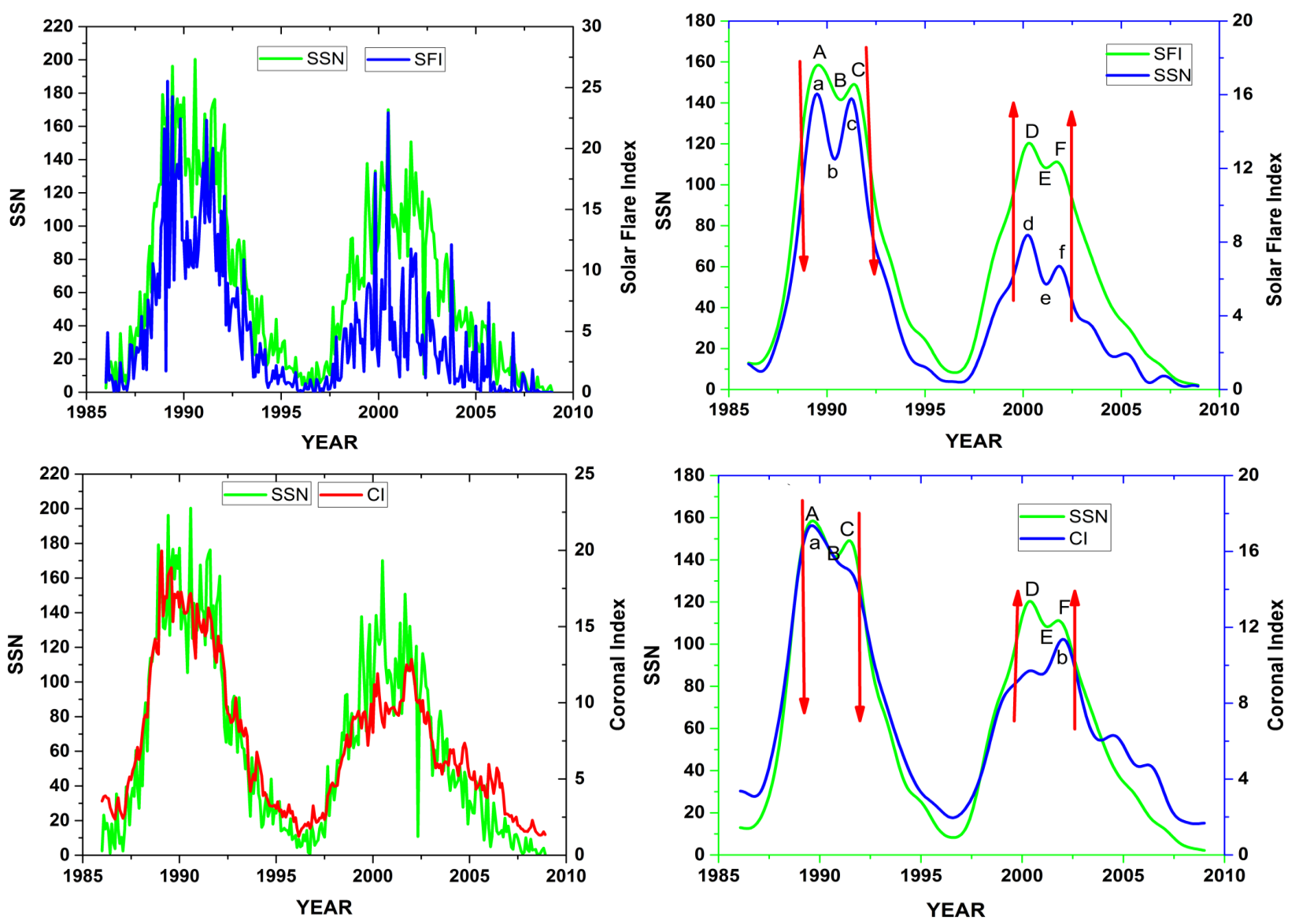

Figure 2. Time profiles of the monthly average values of SSN with CI (upper left panel) and SSN with SFI (lower left panel) and smooth curve ( 6-Months), SSN with CI (upper right panel) and SSN with SFI (lower right panel) during Solar Cycles 22 and 23. 
SFI in solar cycle 22 and at low level of amplitude in cycle 23. Our observation is further support the idea that the solar activity decreases continuously as compared to cycle 22 and 23.

In cycle 22, SSN and SFI reach its first maximum $\sim 3.9$ years after the start. After the first maximum, the SSN and SFI decrease by $14 \%$ (of that maximum). In cycle 23 the SSN and SFI has a first higher maximum flare. This shows that flares can be more efficiently generated solar activity during the first maximum, and it appears that production is declining toward the end of the cycle. Figure 2 shows that for SSN and SFI in the solar activity cycle 23 we can see two maxima separated from each other for about $\sim 1.5$ years. Figure 2 also shows that for SSN and SFI in the solar cycle 23 the relative depth of the amplitude between two maxima is about $10 \%-15 \%$.

\subsection{Correlation Study of CRI with CI and SFI as Well as SSN with CI and SFI}

The trend line of the scattered plot between SSN and CI as shown in the upper left panel of Figure 3, shows a large positive correlation coefficient for solar cycle $22(\mathrm{R}=0.96)$ and solar cycle $23(\mathrm{R}=0.88)$. The correlation coefficient is
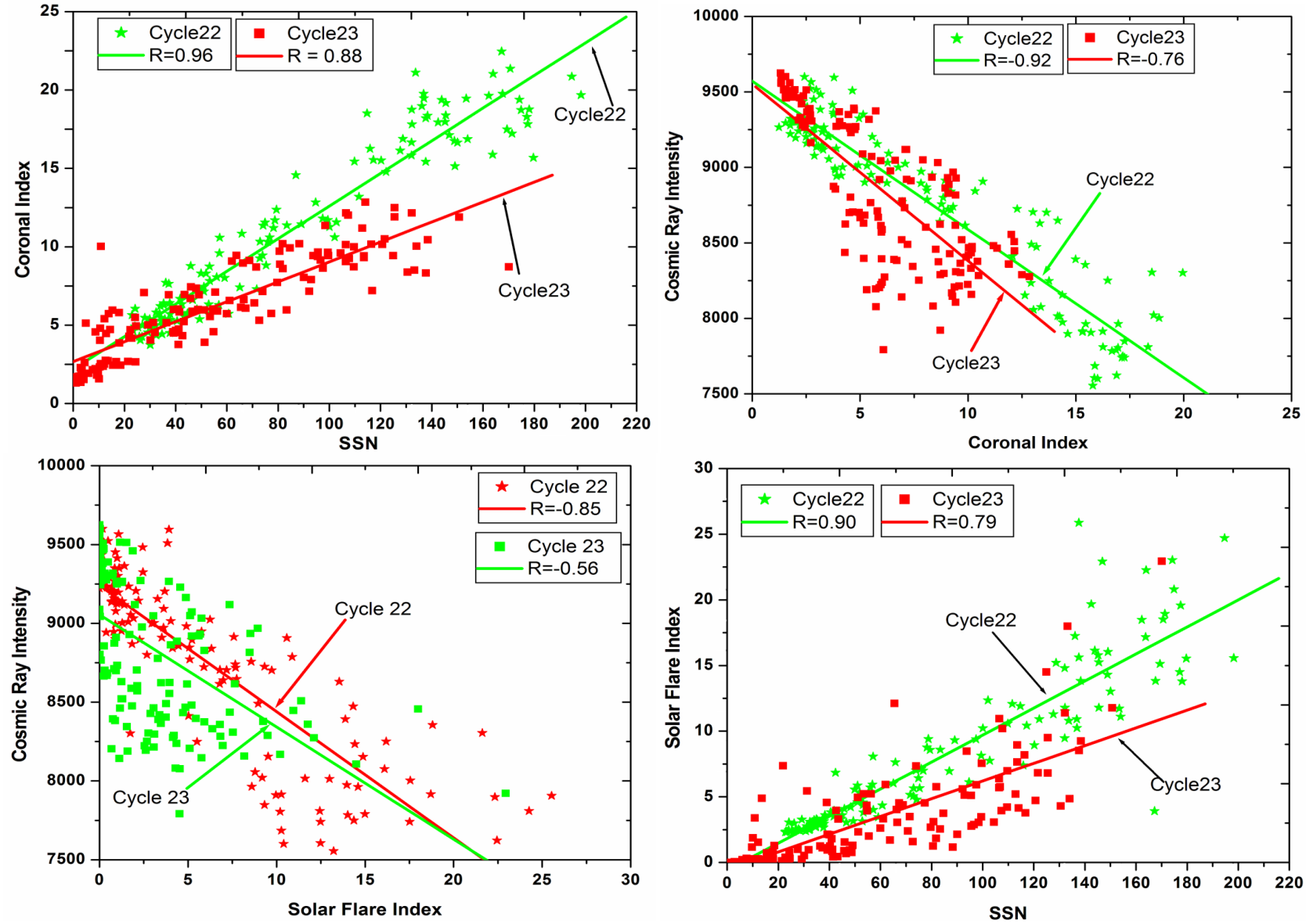

Figure 3. Correlation profiles of the monthly average values of SSN with CI (upper left panel) and SSN with SFI (upper right panel), and CRI with CI (lower left panel) and CRI with SFI (lower right panel) during Solar Cycles 22 and 23. 
decreased in the solar cycle 23 because coronal index of amplitude is decreased. In lower left panel of Figure 3, the trend line of the scattered plot between CI and CRI shows large negative correlation coefficient during solar cycle $22(\mathrm{R}=$ $-0.92)$ and solar cycle $23(\mathrm{R}=-0.76)$ is lowest as compared to previous cycle. The correlation coefficient between SSN and SFI is 0.79 (lowest) for solar cycle 23 and 0.90 (maximum) for solar cycle 22 (see right panel of Figure 3). The peak values of SSN and SFI is found to be continuously decreased for solar cycle 23, so correlation coefficient decreases in solar cycle 23 . The trend line of the scattered plot between SFI and CRI shows large negative correlation for solar cycle $22(\mathrm{R}=-0.85)$ and solar cycle 23 is lowest $(\mathrm{R}=-0.56)$ see left panel of Figure 4. We have observed the regression line (trend line) for solar cycle 23 is quite different for SSN with SFI and CI (see Figure 3), as well as for CRI with CI and SFI (see Figure 3), cross the regression lines (trend line) of solar cycle 22 and attain the lowest position in the graph. Therefore we have, conclude that the correlation coefficient $(\mathrm{R})$ is depending on the solar activity.
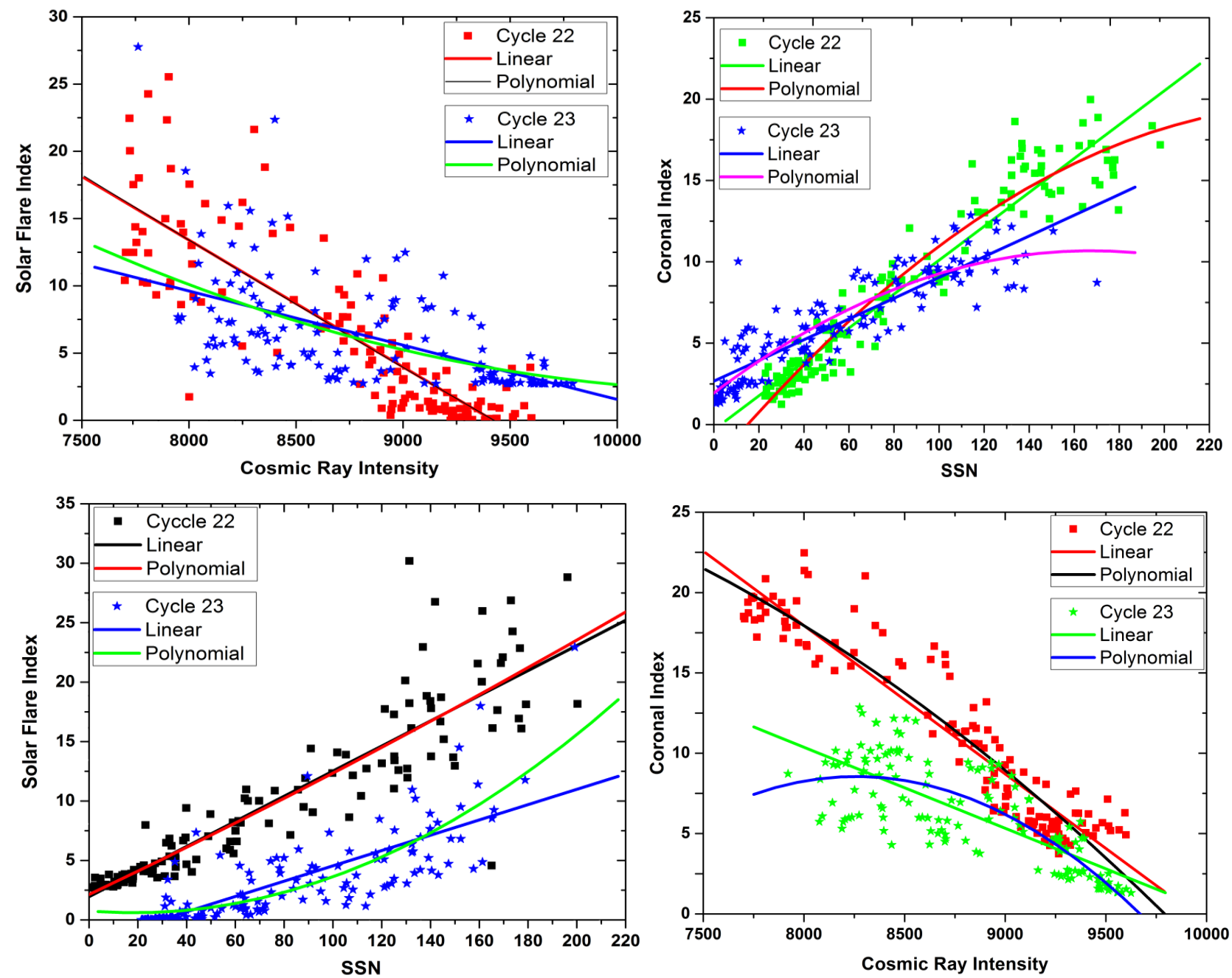

Figure 4. Linear and Polynomial profiles of the monthly average values of CRI with SFI (Top left panel), CRI with CI (Top right panel), and SFI with SSN (bottom left panel), CI with SSN (bottom right panel) during Solar Cycles 22 and 23. 


\subsection{Linear and Polynomial Model of CRI, CI, SSN and SFI}

The linear and polynomial regression lines are continuously tended towards the period 1986-2008. We have noted that the coefficients of linear regression (slope-B and intercept-A) and polynomial regression (intercept-A and coefficients of regression-B1 \& B2) for the solar activity of solar cycles 22 and 23 . We have separated out rise phase, cycle's maximum phase, cycle's minimum phase and decline phase for solar cycles 22 and 23 . We have observed the relation between solar activity as well as cosmic ray intensity; the linear and polynomial regression is quite different for solar cycle 23 to over cycle 22 at attains the lowest position of graph (see Figure 4).

The linear model corresponds to the linear regression equation:

$\mathrm{SFI}=\mathrm{A}+\mathrm{B} . \mathrm{SSN}, \mathrm{SFI}=\mathrm{A}+\mathrm{B} . \mathrm{CRI}$ (Satisfied the straight line equation); and $\mathrm{CI}=\mathrm{A}+\mathrm{B} . \mathrm{SSN}, \mathrm{CI}=\mathrm{A}+\mathrm{B} . \mathrm{CRI}$.

Where $\mathrm{Y}$-is the activity index flux,

$A$ is the intercept of a linear regression,

$B$ Is the slope of a linear regression.

We have found the regression line are significantly different from each other and tend to the $\mathrm{x}$-axis as the slope of the lines (the value of straight line equation, $\mathrm{Y}=\mathrm{B} * \mathrm{X}+\mathrm{A}$ ) decreases continuously during the solar cycles $22 \& 23$ for the period 1986-2008. The standard error is not prominent, for intercept and slope in (SSN with CI), as well as CRI with CI and SFI during the solar cycle $22-23$. The standard error is important for intercept in SSN with SFI. According to our calculations, for linear regression with the highest (positive) values of correlation coefficients (R) for SSN and CI in cycle 22 as well as for CRI and SFI are the minimum (negative) in cycle 23. In the case of linear regression, we have found that the maximum values of the correlation coefficients $(\mathrm{R})$ reached by the phases of rise and fall of the cycles.

The polynomial model corresponds to the following equation of a second order polynomial regression:

$\mathrm{SFI}=\mathrm{A}+\mathrm{B} 1 . \mathrm{SSN}+\mathrm{B} 2 . \mathrm{SSN}^{2}, \mathrm{SFI}=\mathrm{A}+\mathrm{B} 1 . \mathrm{CI}+\mathrm{B} 2 . \mathrm{CRI}^{2} ;$

and $\mathrm{CI}=\mathrm{A}+\mathrm{B} 1 \cdot \mathrm{SSN}+\mathrm{B} 2 \cdot \mathrm{SSN}^{2}, \mathrm{CI}=\mathrm{A}+\mathrm{B} 1 . \mathrm{CRI}+\mathrm{B} 2 \cdot \mathrm{CRI}^{2}$.

Where $\mathrm{Y}$-is the activity index flux,

$A$ is the intercept of a polynomial regression,

$\mathrm{B} 1$ and $\mathrm{B} 2$ are the coefficients of a polynomial regression.

The linear and polynomial regressions presented in Figure 4, the Cosmic Ray Intensity versus SFI and CI (Top panels) and solar activity indices SSN versus SFI and CI (bottom panels) during solar cycles 22 and 23. The Coefficients of linear regressions: A, B and their standard errors $(\sigma)$, for the period 1986-2008 in Table 1. The Coefficients of polynomial regressions: A, B1, B2 and their standard errors $(\sigma)$, for the period 1986-2008 in Table 2.

The standard error is not prominent, for slope in (SSN with CI and SFI), as well as CRI with CI and SFI but the standard error is important for intercept, during the solar cycle $22-23$. We have found that, the polynomial regression for 
Table 1. The Coefficients of linear regressions: A, B and their standard errors $(\sigma)$, for the period 1986-2008.

\begin{tabular}{cccccc}
\hline Act. indices versus SSN & A & B & Err. $\sigma_{\mathrm{A}}$ & Err. $\sigma_{\mathrm{B}}$ & $\mathbf{R}$ \\
\hline $\begin{array}{c}\text { Cycle 22 } \\
\text { Coronal index }\end{array}$ & 1.977 & 0.091 & 0.221 & 0.002 & 0.96 \\
$\mathrm{H}_{\alpha}$ flare index & -0.682 & 0.098 & 0.378 & 0.004 & 0.90 \\
$\quad$ Cycle 23 & & & & & \\
Coronal index & 2.663 & 0.063 & 0.202 & 0.002 & 0.88 \\
$\mathrm{H}_{\alpha}$ flare index & 0.539 & 0.067 & 0.321 & 0.004 & 0.79 \\
\hline Act. indices versus CRI & $\mathrm{A}$ & B & Err. $\sigma_{\mathrm{A}}$ & Err. $\sigma_{\mathrm{B}}$ & $\mathbf{R}$ \\
\hline Cycle 22 & & & & & \\
Coronal index & 89.400 & -0.009 & 2.563 & $2.926 \mathrm{E}-4$ & -0.92 \\
H $\alpha$ flare index & 88.93 & -0.009 & 4.574 & $5.221 \mathrm{E}-4$ & -0.85 \\
$\quad$ Cycle 23 & & & & & \\
Coronal index & 50.734 & -0.005 & 3.118 & $3.526 \mathrm{E}-4$ & -0.76 \\
H $\alpha$ flare index & 42.033 & -0.004 & 4.841 & $5.475 \mathrm{E}-4$ & -0.56 \\
\hline
\end{tabular}

Table 2. The Coefficients of polynomial regressions: A, B1, B2 and their standard errors $(\sigma)$, for the period 1986-2008.

\begin{tabular}{|c|c|c|c|c|c|c|c|}
\hline Act. indices versus SSN & A & B1 & B2 & Err. $\sigma_{\mathrm{A}}$ & Err. $\sigma_{\mathrm{B} 1}$ & Err. $\sigma_{\mathrm{B} 2}$ & $\mathrm{R}^{2}$ \\
\hline \multicolumn{8}{|l|}{ Cycle 22} \\
\hline Coronal index & 1.067 & 0.132 & $-2.363 \mathrm{E}-4$ & 0.280 & 0.008 & $4.969 \mathrm{E}-5$ & 0.92 \\
\hline $\mathrm{H}_{\alpha}$ flare index & -0.505 & 0.090 & $4.583 \mathrm{E}-5$ & 0.519 & 0.016 & $9.201 \mathrm{E}-5$ & 0.81 \\
\hline \multicolumn{8}{|l|}{ Cycle 23} \\
\hline Coronal index & 1.949 & 0.104 & $-3.120 \mathrm{E}-4$ & 0.244 & 0.009 & $6.854 \mathrm{E}-5$ & 0.77 \\
\hline $\mathrm{H}_{\alpha}$ flare index & 0.609 & 0.002 & $4.998 \mathrm{E}-4$ & 0.390 & 0.014 & $1.086 \mathrm{E}-4$ & 0.62 \\
\hline Act. indices versus CRI & A & B1 & B2 & Err. $\sigma_{\mathrm{A}}$ & Err. $\sigma_{\mathrm{B} 1}$ & Err. $\sigma_{\mathrm{B} 2}$ & $\mathrm{R}^{2}$ \\
\hline \multicolumn{8}{|l|}{ Cycle 22} \\
\hline Coronal index & -3.412 & 0.012 & $-1.262 \mathrm{E}-6$ & 46.918 & 0.010 & $6.374 \mathrm{E}-7$ & 0.82 \\
\hline $\mathrm{H}_{\alpha}$ flare index & 100.062 & -0.012 & $1.514 \mathrm{E}-7$ & 84.982 & 0.019 & $1.154 \mathrm{E}-6$ & 0.72 \\
\hline \multicolumn{8}{|l|}{ Cycle 23} \\
\hline Coronal index & -284.859 & 0.071 & -4.300 & 69.454 & 0.015 & $8.892 \mathrm{E}-7$ & 0.58 \\
\hline $\mathrm{H}_{\alpha}$ flare index & 156.301 & -0.030 & $1.464 \mathrm{E}-7$ & 116.046 & 0.026 & $1.485 \mathrm{E}-6$ & 0.42 \\
\hline
\end{tabular}

SSN with CI and SSN with SFI, to value of the intercept is increased as well as value of the slope is decreased during the solar cycles 22 to 23 . The polynomial regression for CRI with SFI the value of the intercept is increased as well as value of the slope is decreased during the solar cycles 22 to 23 . The polynomial regression, for CRI with CI the value of the intercept is decreased as well as the value of the slope is increased for the period 1986-2008. Therefore we have found in the polynomial model the coefficient B2 is not prominent. In the polynomial model the correlation coefficients $\left(R^{2}\right)$ is always positive but decreases during solar cycle $22-23$. On the basics of linear and polynomial model the solar cycle 
23 is lowest amplitude as compare to cycle 22 . We have found solar activity (SSN, SFI and CI) is anti-correlation to CRI by the model of Linear fit.

\section{Conclusions}

We have concluded that the $\mathrm{H}_{\alpha}$ flare index (SFI) and coronal index (CI) are positively correlated with sunspot numbers during solar cycles 22 and 23. The intensity of cosmic ray is inversely correlated with $\mathrm{H}_{\alpha}$ flare index and Coronal Index for the period 1986-2008. $\mathrm{H}_{\alpha}$ flare index and Coronal Index show significantly higher correlation with sunspot number in solar cycle 22 . The correlation coefficient of CRI with the coronal index as well as $\mathrm{H}_{\alpha}$ flare index is relatively large anti-correlation during solar cycle 22 . The correlation coefficient (R) is depending on the solar activity during solar cycle $22-23$. The variation in sunspot number is to be more closely linked with the transient energy in the solar corona. The length of solar cycle 22 is found to be $\sim 9.7$ years whereas $\sim 12.7$ years for solar cycle 23 . Based on the observed results, SFI also seems to be a good representative for the solar index. The long-term modulation of cosmic ray intensity, for solar cycle 23 is characterized by many special features with double peaks. In the long-term smoothed variation by $6^{\text {th }}$ month, for SSN and SFI, many features with double peaks as well as CI are single peak in the each cycle (22 - 23). In this study, we have observed the maximum time-lags between CRI and CI are $\sim 18$ months during solar cycle 23. We have noted the period of Oct. 1999 to Dec. 2003 in SFI with CRI is not prominent (anti-phase) as well as CI with CRI is minimum anti-phase during solar cycle 23 . The above conclusion is that, the solar activities are more dominant for cycle 22 than the cycle 23 as well as solar wind velocity is directly related to the amplitude of the solar activity. The coronal index is more efficiently generated power (irradiance) during the maximum, and it appears that production is declining toward the end of the cycle. The solar activity flare is an important indicator of the energy level of activity of the atmosphere. We have seen two maxima for SSN and SFI, separated from each other for about $\sim 1.5$ years during solar cycle 23 .

The polynomial model is the fittest than the linear fit model, because it gives more accurate correlation coefficient is established for the study period. The model of linear fit says that the solar activity is anti-correlation to the cosmic ray intensity. Therefore, in the polynomial model, the correlation coefficient (positively) is depending on the solar activity. We have concluded the correlation coefficient $(\mathrm{R})$ has the maximum values in the rise and fall phases; the linear relationship between the indices is stronger in these cases. This means that forecasts of SFI and CI, based on SSN and CRI observations will be more successful during the rise and fall of the phase cycle.

\section{Acknowledgements}

The authors are thankful to Dr. C. Vineeth, Scientist (SE), Space Physics Laboratory, Vikram Sarabhai Space Centre (ISRO) Trivandrum (India), for valuable 
suggestions. We sincerely thank an anonymous referee for providing constructive comments and suggestions toward enhancing the quality and presentation of this paper.

\section{References}

[1] Schwabe, H.H. (1843) Die Sonne. Astronomische Nachrichten, 20, 283-286. https://doi.org/10.1002/asna.18430201706

[2] Coles, W.A., Rickett, B.J., Rumsey, V.H., Kaufman, J.J., Turley, D.G., et al. (1980) Solar cycle Changes in the Polar Solar Wind. Nature, 286, 239-241. https://doi.org/10.1038/286239a0

[3] Hathaway, D.H., Wilson, R.M. and Reichmann, E.J. (1999) A Synthesis of Solar Cycle Prediction Techniques. Journal of Geophysical Research, 104, 375-388. https://doi.org/10.1029/1999JA900313

[4] Mishra, A.P., Gupta, M. and Mishra, V.K. (2006) Cosmic Ray Intensity Variations in Relation with Solar Flare Index and Sunspot Numbers. Solar Physics, 239, 475491. https://doi.org/10.1007/s11207-006-0138-x

[5] Choudhuri, A.R. (2011) The Origin of the Solar Magnetic Cycle. PRAMANA Journal of Physics, 77, 77-96. https://doi.org/10.1007/s12043-011-0113-4

[6] Forbush, S.E.J. (1958) Cosmic-Ray Intensity Variations during Two Solar Cycles. Journal of Geophysical Research, 63, 651-669. https://doi.org/10.1029/JZ063i004p00651

[7] Rao, U.R. (1972) Solar Modulation of Galactic Cosmic Radiation. Space Science Review, 12, 719-809. https://doi.org/10.1007/BF00173071

[8] Webber, W.R. and Lockwood, J.A. (1988) Characteristics of the 22-Year Modulation of Cosmic Rays as Seen by Neutron Monitors. Journal of Geophysical Research, 93, 8735-8740. https://doi.org/10.1029/JA093iA08p08735

[9] Aslam, O.P.M. and Badruddin (2012) Solar Modulation of Cosmic Rays during the Declining and Minimum Phases of Solar Cycle 23: Comparison with Past Three Solar Cycles. Solar Physics, 279, 269-288. https://doi.org/10.1007/s11207-012-9970-3

[10] Mishra, A.P., Nigam, S.K. and Singh, R.L. (1992) Propagation Characteristics of Relativistic Solar Cosmic Rays during Current Sunspot Cycle. The Indian Journal of Radio \& Space Physics, 21, 351-353.

[11] Heber, B., Fichtrur, H. and Scherer, K. (2006) Solar and Heliospheric Modulation of Galactic Cosmic Rays. Space Science Reviews, 125, 81-93. https://doi.org/10.1007/s11214-006-9048-3

[12] Chowdhury, P., Kudela, K. and Dwivedi, B.N. (2013) Heliospheric Modulation of Galactic Cosmic Rays during Solar Cycle 23. Solar Physics, 286, 577-591.

https://doi.org/10.1007/s11207-013-0262-3

[13] Singh, P.R., Tiwari, C.M. and Saxena, A.K. (2016) Variations in Solar Cycles 22, 23 \& 24 and Their Effect on Earth's Climate. IJAA, 6, 8-13. https://doi.org/10.4236/ijaa.2016.61002

[14] Carrington, R.C. (1860) Description of a Singular Appearance Seen in the Sun on September 1, 1859. Monthly Notices of the Royal Astronomical Society, 20, 13-15. https://doi.org/10.1093/mnras/20.1.13

[15] Hodgson, R. (1860) On a Curious Appearance Seen in the Sun. Monthly Notices of the Royal Astronomical Society, 20, 15-16. https://doi.org/10.1093/mnras/20.1.15a

[16] Atac, T. and Ozguc, A. (1998) Flare Index of Solar Cycle 22. Solar Physics, 180, $397-$ 
407. https://doi.org/10.1023/A:1005047805338

[17] Ozguc, A. and Atac, T. (2003) Effects of Hysteresis in Solar Cycle Variations between Flare Index and Cosmic Rays. New Astronomy, 8, 745-750. https://doi.org/10.1016/S1384-1076(03)00063-0

[18] Norquist, D.C. (2011) An Analysis of the Sunspot Groups and Flares of Solar Cycle 23. Solar Physics, 269, 111-127. https://doi.org/10.1007/s11207-010-9689-y

[19] Mikic, Z. and Linker, J.A. (1994) Disruption of Coronal Magnetic Field Arcades. The Astrophysical Journal, 430, 898-912. https://doi.org/10.1086/174460

[20] Minarovjech, M. and Kudela, K. (2004) Cosmic Rays and Solar Coronal Emissions: Cross-Correlations of the Time Series. Solar Physics, 224, 285-295. https://doi.org/10.1007/s11207-005-6500-6

[21] Rybansky, M., Rusin, V. and Minarovjech, M. (2001) Coronal Features in the 284A Line and the Polarity of Photospheric Magnetic Fields. Space Science Review, 95, 227-234. https://doi.org/10.1023/A:1005213525647

[22] Xanthakis, J., Petropoulos, B. and Mavromichalaki, H. (1990) Coronal Line Intensity as an Integrated Index of Solar Activity. Astrophysics and Space Science, 164, 117-130. https://doi.org/10.1007/BF00653557

[23] Mavromichalaki, H., Petropoulos, B. and Zouganelis, I. (2002) Long-Term Modulation of Coronal Index of Solar Activity. Solar Physics, 206, 401-414. https://doi.org/10.1023/A:1015091500023

[24] Mavromichalaki, H., Plainaki, C., Zouganelis, I. and Petropoulos, B. (2003) An Empirical Model of the Daily Evolution of the Coronal Index. Solar Physics, 218, 63-78. https://doi.org/10.1023/B:SOLA.0000013050.98809.89

[25] Ahluwalia, H.S. (2003) Solar Wind Modulation of Galactic Cosmic Rays. Geophysical Research Letters, 30, 33. https://doi.org/10.1029/2002GL016017

[26] Badruddin (1996) Cosmic Ray Modulation: Effects of High Speed Solar Wind Streams. Astrophysics and Space Science, 246, 171-191. https://doi.org/10.1007/BF00645638

[27] Bruevich, E.A., Bruevich, V.V. and Yakunina, G.V. (2015) Correlation Study of Some Solar Activity Indices in the Cycles 21-23. arXiv:1304.4545 [astro-ph.SR]

[28] Kleczek, J. (1952) Ionospheric Disturbances and Flares in the 11 years cycle. Bulletin of the Astronomical Institutes of Czechoslovakia, 3, 52.

[29] Ishkov, V.N. (2009) 1st Workshop on the Activity Cycles on the Sun and Stars. Moscow, 18-10 December, 57. 
Submit or recommend next manuscript to SCIRP and we will provide best service for you:

Accepting pre-submission inquiries through Email, Facebook, LinkedIn, Twitter, etc. A wide selection of journals (inclusive of 9 subjects, more than 200 journals)

Providing 24-hour high-quality service

User-friendly online submission system

Fair and swift peer-review system

Efficient typesetting and proofreading procedure

Display of the result of downloads and visits, as well as the number of cited articles Maximum dissemination of your research work

Submit your manuscript at: http://papersubmission.scirp.org/

Or contact ijaa@scirp.org 